\title{
Amplitude variability in $\gamma$ Dor and $\delta$ Sct stars observed by the Kepler spacecraft
}

\author{
Joyce A. Guzik ${ }^{1}$, Katie Kosak ${ }^{1,2}$, Paul A. Bradley ${ }^{1}$ \\ and Jason Jackiewicz ${ }^{3}$ \\ ${ }^{1}$ Los Alamos National Laboratory, Los Alamos, NM USA 87545 \\ email: joy@lanl.gov \\ ${ }^{2}$ Florida Institute of Technology, Melbourne, FL USA 32901 \\ ${ }^{3}$ New Mexico State University, Las Cruces, NM USA 88003
}

\begin{abstract}
The NASA Kepler spacecraft data revealed a large number of multimode nonradially pulsating $\gamma$ Dor and $\delta$ Sct variable star candidates. The Kepler high precision long time-series photometry makes it possible to study amplitude variations of the frequencies. We summarize recent literature on amplitude and frequency variations in pulsating variables. We are searching for amplitude variability in several dozen faint $\gamma$ Doradus or $\delta$ Scuti variable-star candidates observed as part of the Kepler Guest Observer program. We apply several methods, including a Matlab-script wavelet analysis developed by J. Jackiewicz, and the wavelet technique of the VSTAR software (http://www.aavso.org/vstar-overview). Here we show results for two stars, KIC 2167444 and KIC 2301163. We discuss the magnitude and timescale of the amplitude variations, and the presence or absence of correlations between amplitude variations for different frequencies of a given star. Amplitude variations may be detectable using Kepler data even for stars with Kepler magnitude $>14$ with low-amplitude frequencies $(\sim 100 \mathrm{ppm})$ using only one or a few quarters of long-cadence data. We discuss proposed causes of amplitude spectrum variability that will require further investigation.
\end{abstract}

Keywords. stars: $\delta$ Sct; stars: $\gamma$ Dor; stellar pulsations; Kepler spacecraft

\section{Why are amplitude variation unexpected and important?}

For single stars with pulsations unstable to a driving mechanism such as the $\kappa$ mechanism, pulsation properties are determined by the structure of the star, which usually changes very slowly with time via evolutionary processes, e.g., nucleosynthesis for mainsequence stars, or cooling for white dwarfs. The timescales for these processes are hundreds to thousands of years, rather than the hours to years over which we have photometric data and detect significant variations. Since growth rates calculated by linear nonadiabatic pulsation codes can be large (normalized work $10^{-3}-10^{-6}$ per period), pulsation amplitudes should grow relatively quickly to reach a limiting amplitude. On the other hand, stochastically excited pulsations, as found in solar-like and red giant stars, are continuously excited and damped, so their amplitudes are expected to vary.

Amplitude variations may be useful diagnostics of energy partition/exchange between modes, and may involve modes of degree $l \geqslant 3$ that are more difficult to observe in photometry, or gravity modes with high amplitude in the stellar interior that aren't visible at the surface, or even stable modes. These variations may tell us something about energy exchange with internal dynamical processes (convection, rotation, magnetic fields) or changes in ionization regions that we cannot observe directly. They may indicate interaction with the external environment, e.g., via mass outflow, accretion, or tidal forces from a binary companion or planet. Understanding the mechanisms that limit amplitudes 

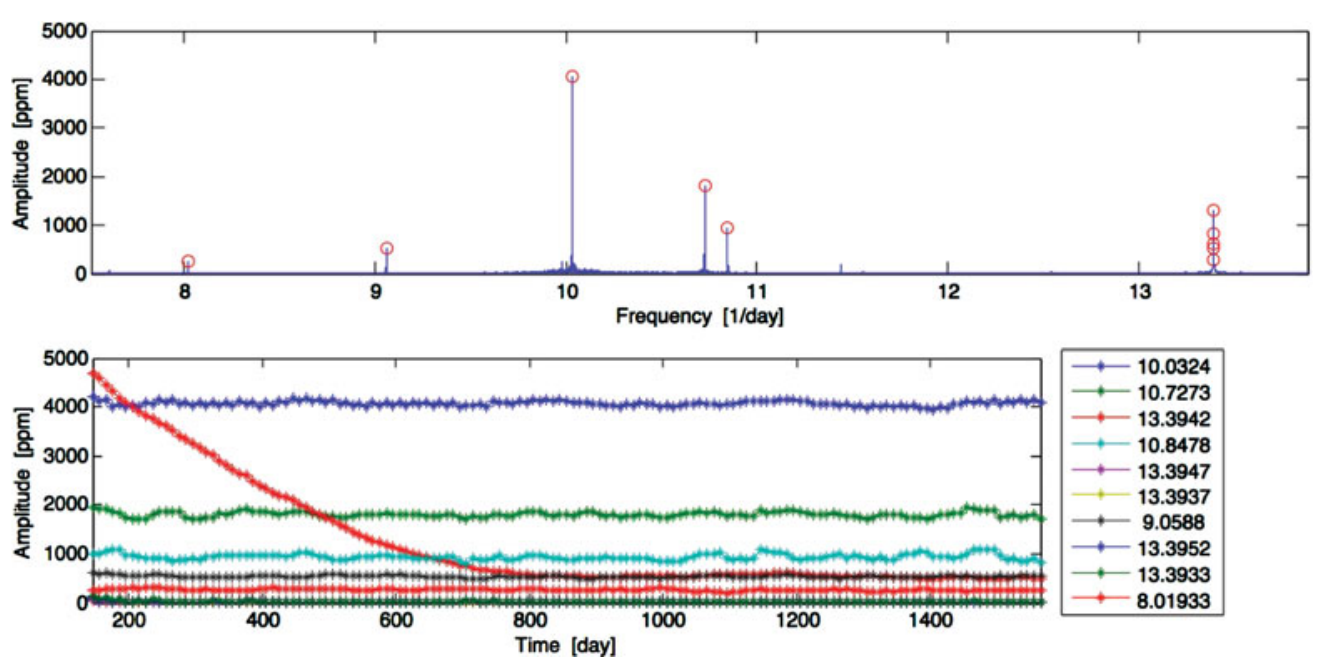

Figure 1. Matlab-script generated KIC 7106205 amplitude spectrum (top) and amplitude vs. time for ten highest-amplitude modes. The mode at $13.3942 \mathrm{c} / \mathrm{d}$ shows a dramatic amplitude decrease during the first $\sim 600$ days, as first reported by Bowman \& Kurtz (2014).

or cause amplitude variations will be important to understand mode selection, and to validate nonlinear nonradial and nonadiabatic pulsation models.

\section{Causes of amplitude/frequency variations}

Amplitude and/or frequency variations have been found among nearly all types of nonstochastically excited pulsating variables: $\delta$ Sct (Breger et al. 2012, Breger \& Pamyatnykh 2006, Bowman \& Kurtz 2014); $\gamma$ Dor (Rostopchina et al. 2013); $\beta$ Cep (Pigulski \& Pojmanski 2008), roAp (Balona et al. 2013, Medupe et al. 2015); classical pulsators such as high-amplitude $\delta$ Sct stars (Zhou \& Jiang 2011, Khokhuntod et al. 2011), Cepheids (Engle 2015), RR Lyrae (Chadid \& Preston 2013), Miras and yellow supergiants (Percy \& Yook 2014, Percy \& Khatu 2014); white dwarfs (DBV; Handler et al. 2003 and DAV; Bell et al. 2015); GW Vir stars (Vauclair et al. 2012), sdB stars (Kilkenny 2010, Langfellner et al. 2012), and an extreme helium subdwarf (Bear \& Soker 2014).

A non-comprehensive list of proposed explanations for the variations includes: parametric instability (unstable high frequency mode $\nu_{1}$ excites two lower frequency stable modes with $\nu_{2}+\nu_{3}=\nu_{1}$; see Dziembowski \& Krolikowska 1985); resonant mode coupling (Barceló Forteza et al. 2015); stochastic excitation instead of intrinsically unstable modes (see, e.g., Huber et al. 2011); energy from the modulated mode exchanged with, e.g., the convection zone, ionization region or magnetic field; 'weather' (atmospheric disturbances) from a tidally locked planet (Bear \& Soker 2014); tidal effects from an unseen binary or planetary companion; outbursts (e.g., Be star 102719279; Gutierrez-Soto et al. 2010), or accretion (e.g., GW Lib; Toloza et al. 2015) changing the star's structure; pulsations sampling the crystallization region (white dwarf interior; Hermes et al. 2015); diffusive settling of helium (Cox 1998); the star is caught during a phase of rapid evolution, e.g., at the edge of an instability strip, or during the rapid core contraction phase at the end of core hydrogen burning. Sometimes apparent amplitude variations are attributable to insufficiencies in the time series data or analysis, e.g., to very close frequencies not being resolved, an interruption in the time series, or an artifact from the temporal distribution of data. The papers by Bowman \& Kurtz (2014), Breger \& Montgomery (2014), 

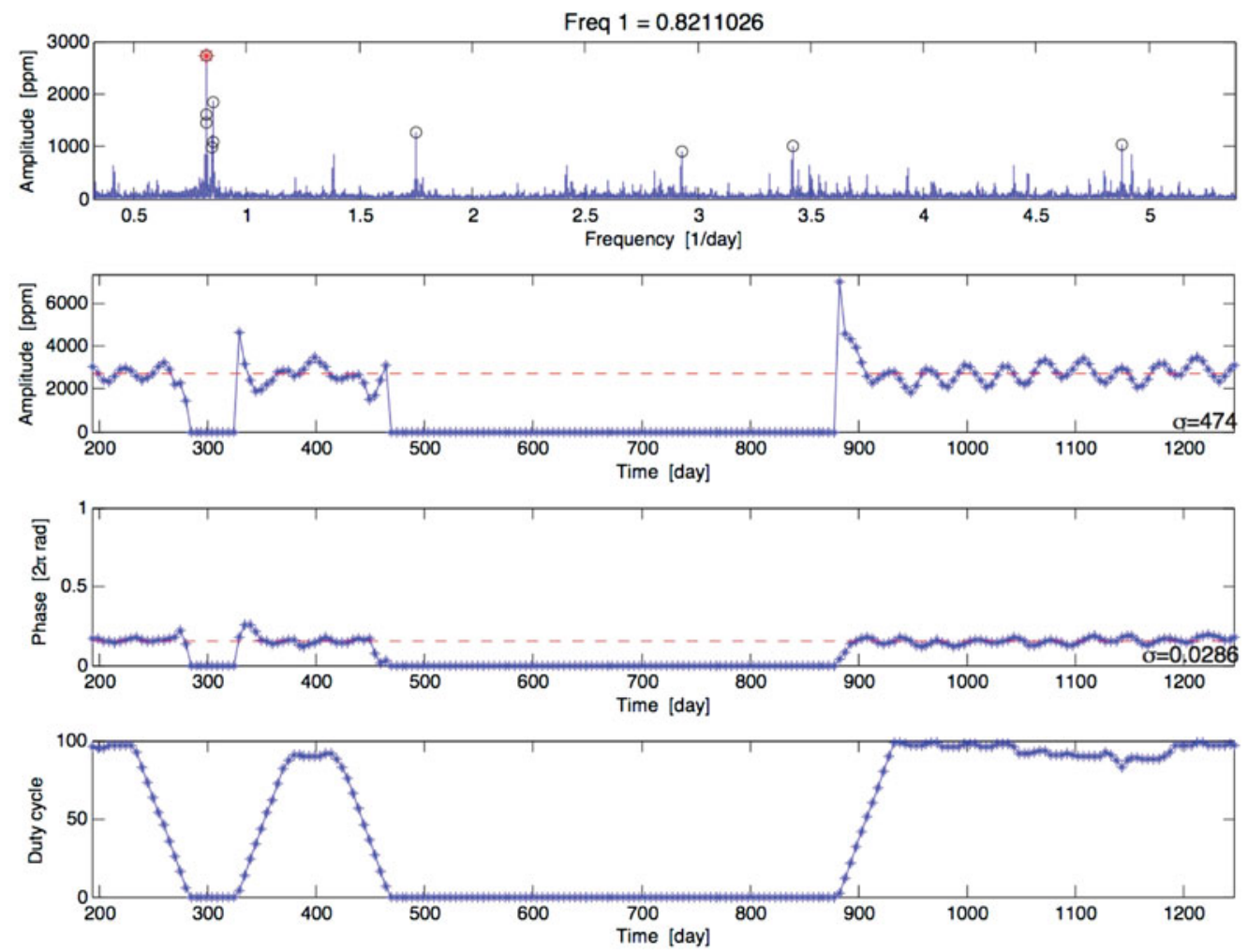

Figure 2. Matlab-script amplitude spectrum (top panel) and wavelet analysis for amplitude (second panel) and phase (third panel) variations, and duty cycle (bottom panel) for highest amplitude $0.8211 \mathrm{c} / \mathrm{d}$ mode for KIC 2167444. Although we have applied a correction for decreasing duty cycle, the amplitudes are still unreliable very near the time regions where the duty cycle decreases to zero. The amplitude and phase variations show the same periodicity but are offset in phase by $\sim 90^{\circ}$, as is expected for beating between two modes according to Breger \& Pamyatnykh (2006).

Barceló Forteza et al. (2015), Holdsworth et al. (2014), and Percy \& Khatu (2014) provide discussion and further references for causes of amplitude and frequency variations.

\section{Amplitude variations in $\gamma$ Dor $/ \delta$ Sct stars}

Detecting (or ruling out) amplitude variations requires high-precision continuous time series data that captures many pulsation periods. We now have many data sets that can be used to study such variations, including those from Kepler and CoRoT $(\delta$ Sct and $\gamma$ Dor stars), ASAS (B stars), WET (white dwarfs and roAp stars), and AAVSO (longperiod variables and giants) observations. The Kepler spacecraft has returned continuous time-series observations spanning months to years, either in long cadence (30-minute integrations per data point), or short cadence (1-minute integrations), with micro-magnitude precision in the amplitude spectrum.

We are searching for amplitude variations in mostly faint $\left(\mathrm{K}_{p} \operatorname{mag}>14\right) \gamma$ Dor and $\delta$ Sct candidates discovered in long-cadence data via the Kepler Guest Observer program. We used the weighted-wavelet z-transform technique (Foster 1996) available in the VSTAR software from the American Association of Variable Star Observers. For the example analyses shown below, we use $\sim 1000$ data points per Gaussian wavelet, that translate to 

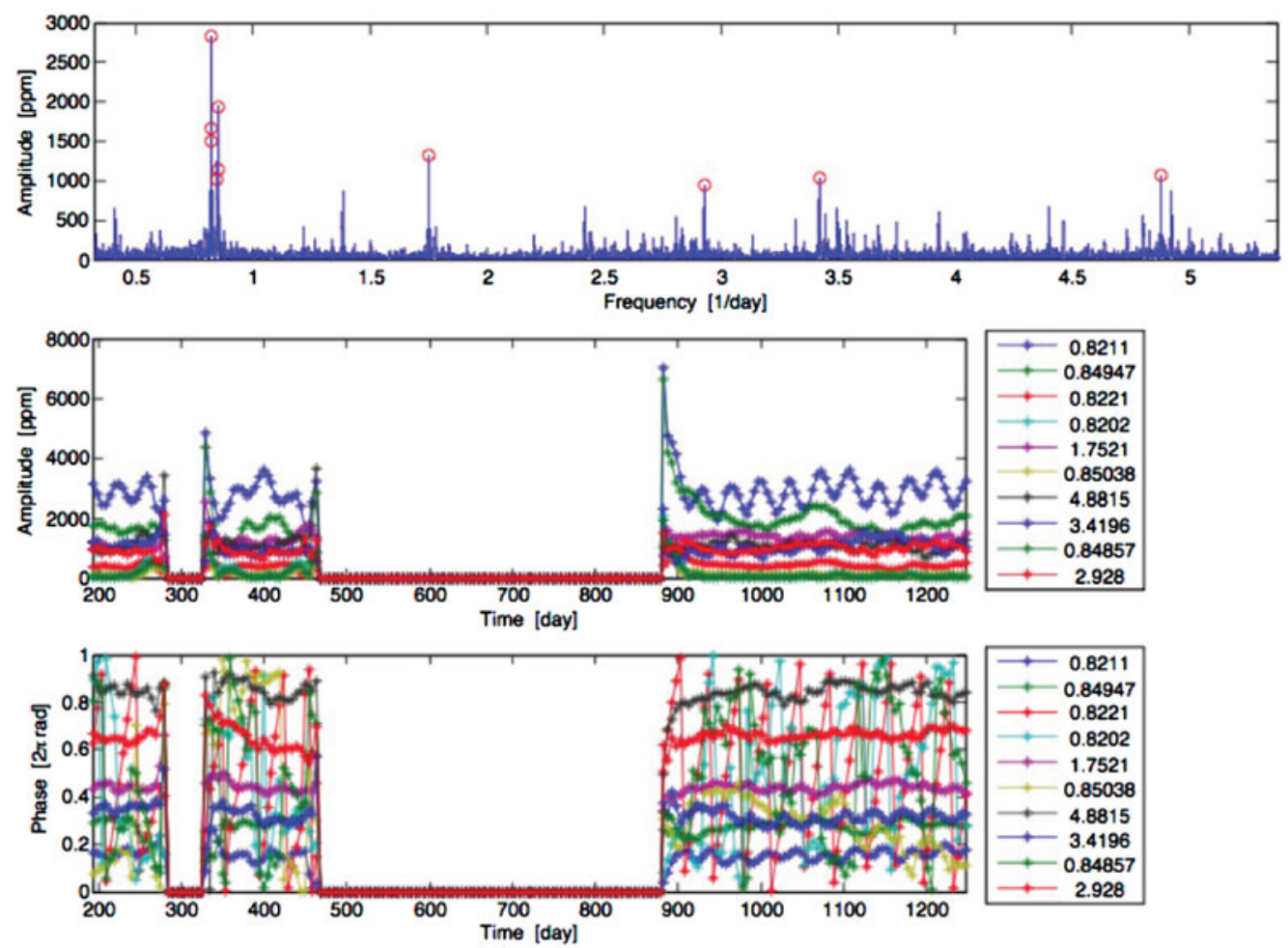

Figure 3. Matlab-script amplitude spectrum (top panel) and wavelet analysis for amplitude variation (middle panel) and phase variation (bottom panel) of 10 highest-amplitude modes of KIC 2167444. The four modes with rapid phase variation are spaced about $0.001 \mathrm{c} / \mathrm{d}$ from the main peaks at $0.8211,0.8495 \mathrm{c} / \mathrm{d}$ main peak, and are likely artifacts of the evenly spaced 30 -minute cadence light curve that is oversampled by a factor of 10 .
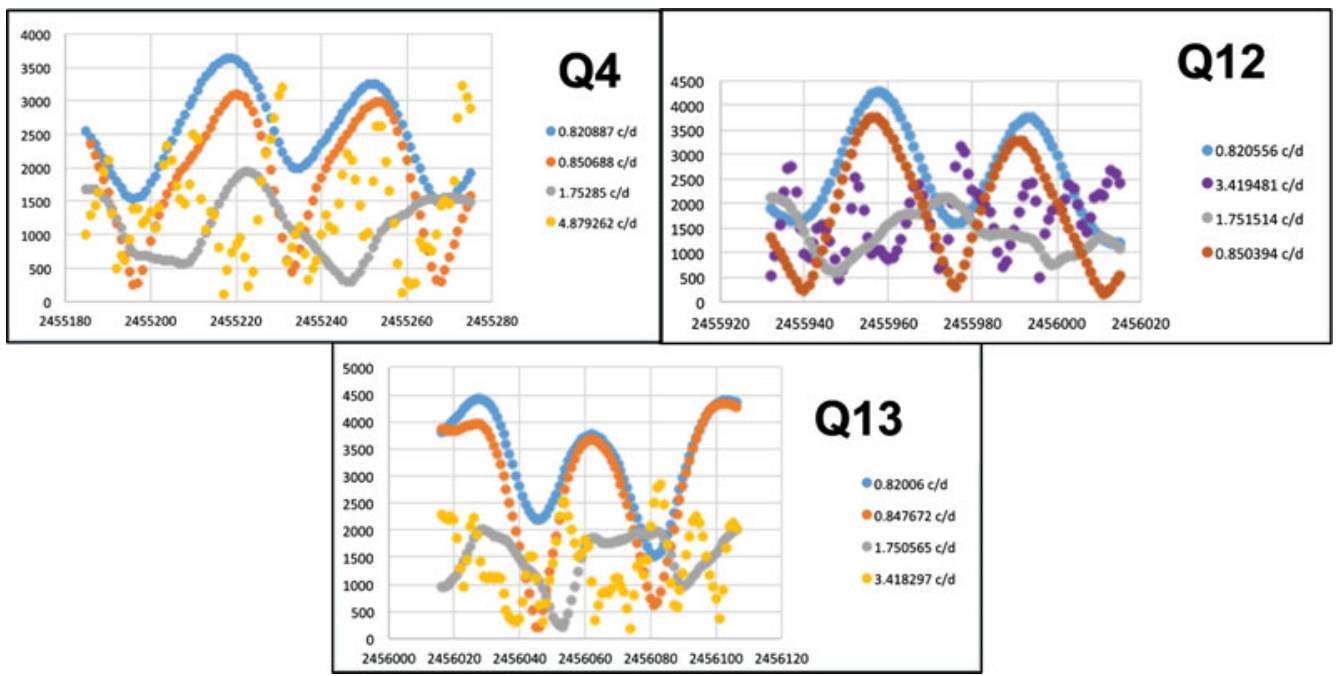

Figure 4. VSTAR wavelet analysis for KIC 2167444 Q4, Q12, and Q13 data. The highest-amplitude modes at 0.821 (blue dots) and 0.851 (orange dots) c/d show $\sim 35$-day amplitude modulations that are not exactly in phase with each other. The next two highest-amplitude modes show quasi-periodic amplitude variations. 

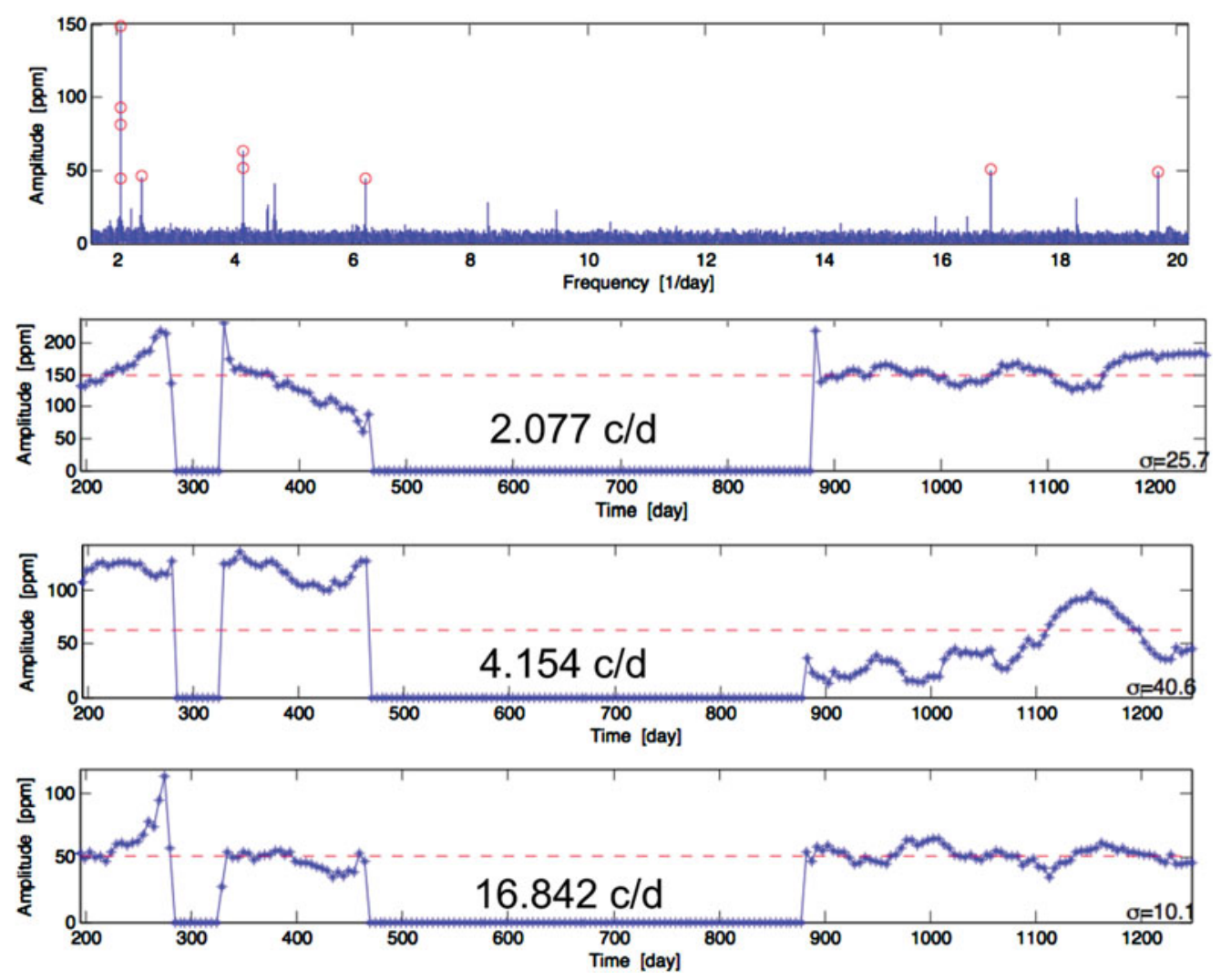

Figure 5. Matlab-script amplitude spectrum (top panel) and wavelet analysis (next three panels) for highest-amplitude modes of KIC 2301163 . The $2.077 \mathrm{c} / \mathrm{d}$ mode and its possible harmonic at $4.154 \mathrm{c} / \mathrm{d}$ do not show the same amplitude variation. The $\delta$ Sct-type p mode at $16.842 \mathrm{c} / \mathrm{d}$ does not show significant amplitude variation.

$\sim 20$-day windows for long-cadence data. We also show results for the wavelet-analysis script written using Matlab by J. Jackiewicz, choosing 50-day wide data windows, with 10-day or 5-day offsets. We note that it is important to consider the width of the time series to analyze, as well the offset between windows; too-large windows and too-large offsets will average out or decrease the size of amplitude variations, and too-small windows will have low signal-to-noise and fail to resolve closely spaced modes.

We first tested the Matlab script on Kepler data for KIC $7106205\left(\mathrm{~K}_{p} \operatorname{mag}=11.455\right)$, and easily detect in long-cadence data the large amplitude decrease of the $13.3942 \mathrm{c} / \mathrm{d}$ mode reported by Bowman \& Kurtz (2014) (Fig. 1). This script corrects the amplitude and phase vs. time for reduced duty cycle in the Kepler data. As seen in later analysis, this correction is only partially successful at the edges of observation quarters without data (zero duty cycle).

\section{1. $K I C 2167444$}

KIC 2167444 is a $\mathrm{K}_{p}$ mag $14.1 \gamma$ Dor candidate observed by Kepler in long cadence during Quarters 2, 4, and 10-13. Figure 2 shows the Matlab-script amplitude spectrum (top panel) and the wavelet-analysis amplitude (2nd panel) and phase (3rd panel) vs. time for the highest-amplitude peak at $0.8211 \mathrm{c} / \mathrm{d}$. The amplitude and phase show a pronounced modulation of about 32 days. The amplitude is not reliable in the regions where the duty cycle drops off quickly (bottom panel). Figure 3 shows the Matlab-script 
results for the amplitude and phase variations of the 10 highest-amplitude modes (marked with red circles on the amplitude spectrum in the top panel). The second-highest amplitude mode at $0.8495 \mathrm{c} / \mathrm{d}$ shows an amplitude modulation similar to the $0.8211 \mathrm{c} / \mathrm{d}$ mode during Q2 and 4, but a more gradual variation in Q10-13. These two close frequencies should be resolved with the 50-day wavelet window, (resolution $0.02 \mathrm{c} / \mathrm{d}$ ); their beating frequency is $(1 /(0.8211025-0.8494719))=\sim 35$ days, so it is possible that beating between these modes is causing the amplitude variation, or that these two modes are interacting. There is a phase shift of $\sim 90^{\circ}$ between the amplitude and phase variation of this mode, which fulfills the criteria for two modes beating against each other, according to Breger \& Pamyatnykh (2006). The modes at 1.7521, 4.8815, 3.4196, and $2.9280 \mathrm{c} / \mathrm{d}$ also show more subdued amplitude variations with similar periodicity; these modes are not obvious combination frequencies of the two highest-amplitude modes, so the periodicity is puzzling. An alternative explanation is that several highamplitude modes are coupling to a low-frequency, possibly stable mode, as suggested by Barceló Forteza et al. (2015) to explain amplitude modulations in the $\delta$ Sct star KIC 5892969.

The pair of modes at 0.8221 and $0.8202 \mathrm{c} / \mathrm{d}$, and the pair at 0.8486 and $0.8504 \mathrm{c} / \mathrm{d}$ show very large phase variations in Fig. 3 (bottom panel). These modes are all multiples of $\sim 0.001 \mathrm{c} / \mathrm{d}$ away from a main mode, and are likely side-lobe artifacts generated by the finite-length data set of 1050 days $(1 / 1050$ days $=\sim 0.001 \mathrm{c} / \mathrm{d})$. These modes essentially disappear when the main mode is prewhitened.

We also examined the data using the VSTAR wavelet analysis, and show results for the four highest-amplitude modes in Quarters 4, 10, and 13 (Fig. 4). VSTAR finds the same highest-amplitude modes, including the 0.82 and $0.85 \mathrm{c} / \mathrm{d}$ modes. The VSTAR plots show that the amplitude variations between the 0.82 and $0.85 \mathrm{c} / \mathrm{d}$ highest amplitude modes are not exactly in phase with each other. The amplitude variation of the $1.75 \mathrm{c} / \mathrm{d}$ mode is more regular during Q4 than in subsequent quarters. In Q12 and 13, the $3.42 \mathrm{c} / \mathrm{d}$ mode has a higher amplitude, while in $\mathrm{Q} 4$ the $4.88 \mathrm{c} / \mathrm{d}$ mode is more prominent.

\section{2. $K I C 2301163$}

KIC 2301163 is a $K_{p}$ mag $14.1 \gamma$ Dor $/ \delta$ Sct hybrid candidate that was also observed in Quarters 2, 4, and 10-13. This star shows significant amplitude variations, even though the amplitudes are an order of magnitude lower than for KIC 2167444 ( 100 ppm instead of $\sim 1000 \mathrm{ppm}$ ). Figure 5 shows the amplitude spectrum and the amplitude vs. time for three of the highest-amplitude peaks. The highest-amplitude mode at $2.077 \mathrm{c} / \mathrm{d}$ and the next-highest amplitude mode at $4.154 \mathrm{c} / \mathrm{d}$, nearly exactly twice the frequency of the first mode, show different amplitude variations, even though one mode could be a harmonic of the other. The possible $\delta$ Sct-type p mode at $16.842 \mathrm{c} / \mathrm{d}$ does not show significant amplitude variation (standard deviation $10 \mathrm{ppm}$, about the noise level of the amplitude spectrum).

\section{Conclusions}

Amplitude variations may be detectable by Kepler even for stars with $\mathrm{K}_{p}>14$ with lowamplitude frequencies $\sim 100 \mathrm{ppm}$ using only one or a few quarters of long-cadence data. Amplitude variations for different frequencies are sometimes correlated. It seems that analysis of the Kepler data to interpret amplitude variations requires significant effort for each star, and that it will be difficult to find patterns or draw a general conclusion by studying an ensemble of stars. 


\section{Acknowledgements}

We gratefully acknowledge the NASA Kepler Guest Observer Program, the DOE Science Undergraduate Laboratory Internship program, and Los Alamos National Laboratory.

\section{References}

Balona, L. A., et al. 2013, MNRAS, 432, 2808

Barceló Forteza, B., et al. 2015, A\& $A, 579$, A133

Bear, E. \& Soker, N. 2014, MNRAS, 437, 1400

Bell, K. J., et al. 2015, ApJ, 809, 14

Bowman, D. \& Kurtz, D. 2014, MNRAS, 444, 209

Breger, M., et al. 2012, ApJ, 759, 62

Breger, M. \& Montgomery, M. 2014, ApJ, 783, 89

Breger, M. \& Pamyatnykh, A. A. 2006, MNRAS, 368, 571

Chadid, M. \& Preston, G. W. 2013, EAS, 63, 47

Cox, A. N. 1998, ApJ, 496, 246

Dziembowski, W. \& Krolikowska, M. 1985, AcA, 35, 5

Engle, S. G. 2015, Ph.D. Thesis, James Cook University, 2015arXiv150402713E

Foster, G. 1996, AJ, 112, 1709

Gutierrez-Soto, J., et al. 2010, 2010arXiv1010.1910G

Handler, G., et al. 2003, MNRAS, 340, 1031

Hermes, J. J., et al. 2015, ASPC, 493, 59

Holdsworth, D. L., et al. 2014, MNRAS, 443, 2049

Huber, D., et al. 2011, ApJ, 743, 143

Khokhuntod, P., et al. 2011, IBVS, in preparation, 2011arXiv1109.3840K

Kilkenny, D. 2010, Ap\&SSS, 329, 175

Langfellner, J., et al. 2012, ASPC, 452, 203

Medupe, R., et al. 2015 MNRAS, 446, 1347

Percy, J. R. \& Khatu, V. C. 2014, JAAVSO, 42, 1

Percy, J. R. \& Yook, J. Y. 2014, JAAVSO, 42, 245

Pigulski, A. \& Pojmanski, G. 2008, A\&A, 477, 907

Rostopchina, A., Breger, M., \& Hansen, G. 2013, AAS, 22135424R

Toloza, O., et al. 2015, ASPC, 493, 253

Vauclair, G., et al. 2012, ASPC, 462, 160

Zhou, A.-Y. \& Jiang, S.-Y. 2011, AJ, 142, 100 